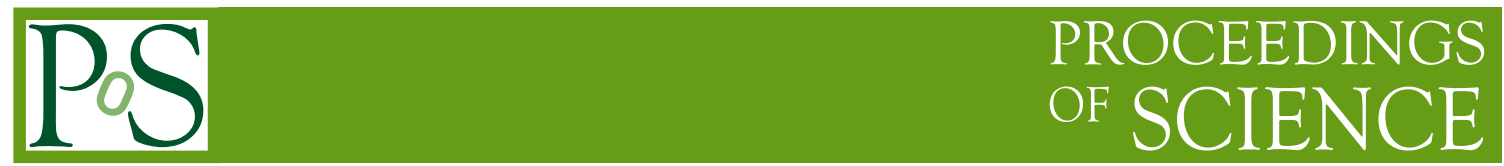

\title{
Central Exclusive Production at LHCb
}

\author{
Bartłomiej Rachwał* \\ AGH-UST University of Science and Technology, Faculty of Physics and Applied Computer \\ Science, Krakow, Poland \\ E-mail: brachwaleagh.edu.pl
}

The LHCb detector, with its excellent momentum resolution and flexible trigger strategy, is ideally suited for measuring particles produced exclusively. In addition, a new system of forward shower counters has been installed upstream and downstream of the detector, and has been used to facilitate studies of Central Exclusive Production. Such measurements of integrated and differential cross-section in both Run 1 and Run 2 of the LHC, are summarised here.

XXV International Workshop on Deep-Inelastic Scattering and Related Subjects 3-7 April 2017

University of Birmingham, $U K$

${ }^{*}$ Speaker. 


\section{Introduction}

The theme of these proceedings is central exclusive particle production (CEP) in $p p$ collisions studied at LHCb experiment [1]. In these interactions particles are produced in between two large rapidity gaps, while the incident beam particles either stay intact or dissociate to a cluster of hadrons. Generically one can write $p p \rightarrow p(*)+X+p(*)$, where " $+"$ signs denote the presence of large rapidity gaps, separating the system $X$ from the outgoing protons [2]. The central system $X$ of created particles is fully measured (i.e. exclusive).

The dynamics of the CEP process leads to unique predictions and effects which are not seen in inclusive modes. In general terms the study of CEP requires an explicit account of both soft and hard Quantum Chromodinamics (QCD), and is therefore sensitive to both of these regimes. Experimentally, CEP represents a very clean signal, with just the object $X$ and no other hadronic activity seen in the central detector (in the absence of pile up).

One can find the measurements of exclusive processes can be performed by installing proton tagging detectors, situated far down the beam line from the central detector, which can provide information about the mass and quantum numbers of the centrally produced state. However, such measurements can be performed both with and without tagged protons at low to medium luminosity during general LHCb runs.

\section{Measuring exclusivity with $\mathrm{LHCb}$}

The LHCb detector $[1,3]$ is fully instrumented for pseudorapidities $2<\eta<4.5$, and includes a high-precision tracking system of a silicon-strip vertex detector (VELO) surrounding the $p p$ interaction region, which has sensitivity to charged particles in the backwards region, as well as in the forward region. To increase the rapidity coverage of the LHCb spectrometer, forward shower counters consisting of five planes of scintillators (HeRSCheL) at $-114,-19.7,-7.5,+20$ and $+114 \mathrm{~m}$ from the interaction point have been installed for Run 2, which started in 2015 [3]. In summary, the combination of VELO and HeRSCheL has sensitivity to particles in the regions $-10<\eta<-5,-3.5<\eta<-1.5,1.5<\eta<10$. Thus the Run 1 rapidity coverage sums to roughly 5.5 units in rapidity while the Run 2 to 12 units [2].

The selection of events which are dominantly due to CEP is performed using rapidity gap methods, i.e. requiring that there is no additional hadronic activity associated with the event in a large enough region of pseudorapidity. Moreover, to cope with quasi-exclusive interactions containing low mass proton dissociation, a wide rapidity coverage of veto detectors is advantageous. The unique forward coverage of the LHCb detector is well suited for such analysis.

The CEP analysis techniques are based on vetoing an additional activity in the detector and require the probability of more than one proton interaction per bunch crossing to be small. This number of visible $p p$ interactions per beam crossing, $N$, is assumed to follow a Poisson distribution: $\mathscr{P}(N)=e^{\mu} \mu^{N} / N$ !, where $\mu$ is the average number of visible interactions. The summary of LHCb datasets used in the described here analyses, together with an averaged over the data-taking period, the probability for a single interaction is given in Tab. 1. The experiment is operated at a low number of interactions per beam crossing since the beams are partially defocused close to LHCb. The analyses presented below are based on data from both Run 1 and 2 periods. 
Table 1: The LHCb datasets and corresponding parameters described in text.

\begin{tabular}{ccccc} 
Year & CMS energy $[\mathrm{TeV}]$ & $\mathscr{L}\left[\mathrm{fb}^{-1}\right]$ & $\mu$ & $\mathscr{P}(1)[\%]$ \\
\hline \hline 2011 & 7.0 & 1 & 1.4 & 25 \\
2012 & 8.0 & 2 & 1.7 & 19 \\
2015 & 13.0 & 0.2 & 1.08 & 35
\end{tabular}

Figure 1 shows the discrimination power of the CEP analysis techniques. On the left plot of Fig. 1 the distribution of tracks in the forward region is presented which is affected by backward rapidity gap required; a clear peak is visible at precisely two forward tracks which are candidates for exclusive production. The ability of HeRSCheL to discriminate CEP events corresponds to significant energy deposits in HeRSCheL quantified as quadratic sum, $\Sigma_{H}$, of the normalised signals in each of the 20 channels:

$$
\Sigma_{H}=\sum_{n=1}^{20}\left(\frac{A D C_{i}}{2.5 R M S_{i}}\right)^{2} .
$$

The distributions of $\Sigma_{H}$ included in the right plot of Fig.1 are related to three classes of lowmultiplicity-triggered events: (i) the non-resonant dimuon sample (electromagnetic CEP events), (ii) the inelastic events with proton dissociation, (iii) events with more than five reconstructed tracks. The CEP enriched events have lower values of $\Sigma_{H}$, thus events are removed by requiring that $\Sigma_{H}$ is lower than a given threshold.
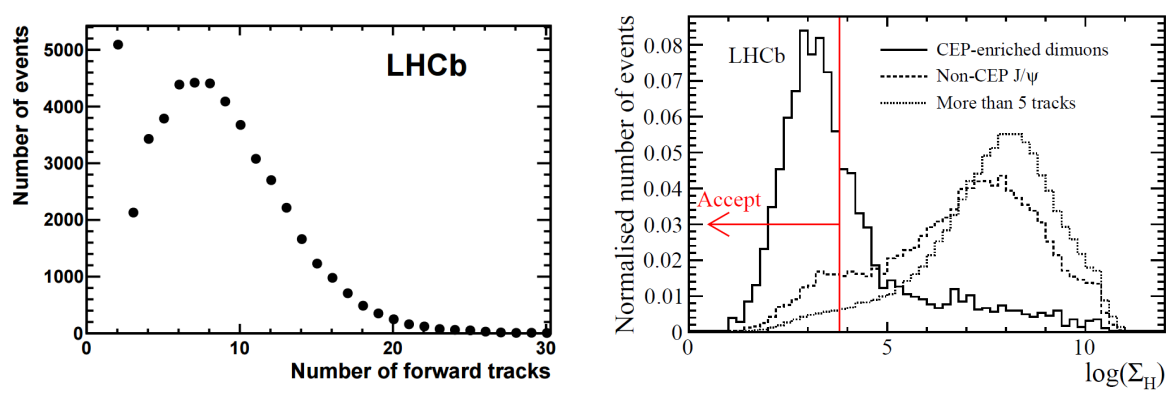

Figure 1: (Left) Number of tracks in the forward region for dimuon triggered events with the backward tracks veto being required, from [4]. (Right) The HeRSCheL discriminant based on the detector acttivity enhanced in the formula 2.1, from [6].

\section{CEP measurements at $\mathrm{LHCb}$}

The approach taken in the LHCb analyses to date is to invoke Regge theory to describe the shape of the transverse momentum squared $\left(p_{\mathrm{T}}^{2}\right)$ distribution. The $p_{\mathrm{T}}^{2}$ spectrum is fit with different components to separate the elastic CEP process from backgrounds, extracting the signal fraction. This method assumes that each component can be modelled by an exponential function.

\section{1 $\mathrm{J} / \psi$ and $\psi(2 S)$ meson production}

The measurements of exclusive $\mathrm{J} / \psi$ and $\psi(2 S)$ production have been performed at $\mathrm{LHCb}$ based on data collected at $\sqrt{s}=7 \mathrm{TeV}[4,5]$ and $\sqrt{s}=13 \mathrm{TeV}$ [6], cf. Tab.1. Events are selected 
with two muons and no other track in the VELO. Furthermore, for the later analysis the HeRSCheL veto is implied, which allows backgrounds to be significantly reduced with respect to previous measurement made at lower energy.

In the $\sqrt{s}=7 \mathrm{TeV}$ analysis, three components are included in the fit: an exponential for elastic CEP, an exponential for the inelastic background, and the feed-down (from exclusive production of other mesons) component from data, while the $\sqrt{s}=13 \mathrm{TeV}$ data are fit by two exponential functions, where one describes signal and one the proton dissociation component. In the latter case the subtraction of the non-resonant and feed-down contributions are already applied. Fig.2 shows the fits performed for $\mathrm{J} / \psi$ candidates for both measurements described in this proceedings.
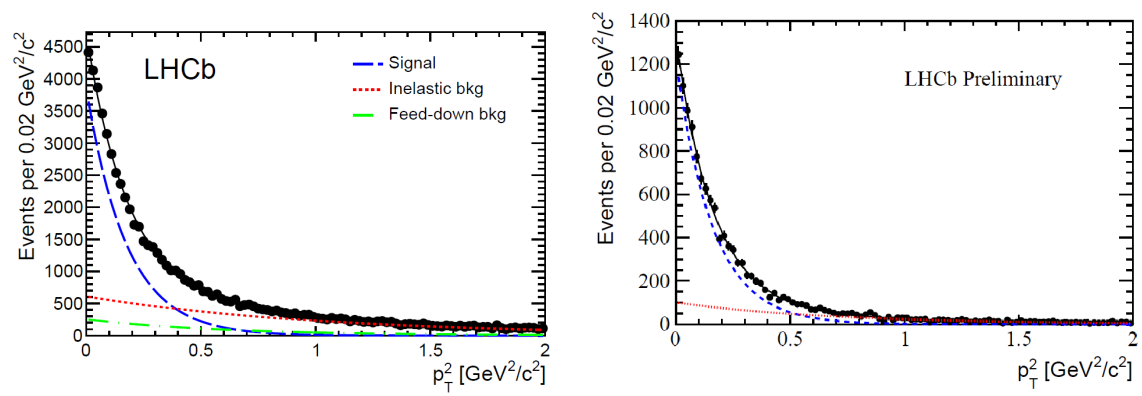

Figure 2: Transverse momentum squared of $\mathrm{J} / \psi$ candidates for (left) $\sqrt{s}=7 \mathrm{TeV}$ and (right) $\sqrt{s}=13 \mathrm{TeV}$ data, from $[4,6]$. The fitted contributions details are described in text.

The efficiencies for tracking, muon identification and triggering are determined from data, while the selection efficiency is determined from simulation. The cross-section times branching fractions for the decays to dimuons, where both muons are within the pseudorapidity region $2<$ $\eta<4.5$, are found to be $\sigma_{\mathrm{J} / \psi \rightarrow \mu^{+} \mu^{-}}=291.0 \pm 7 \pm 19 \mathrm{pb}, \sigma_{\psi(2 S) \rightarrow \mu^{+} \mu^{-}}=6.5 \pm 0.9 \pm 0.4 \mathrm{pb}$ and $\sigma_{\mathrm{J} / \psi \rightarrow \mu^{+} \mu^{-}}=407.0 \pm 8 \pm 24 \pm 16 \mathrm{pb}, \sigma_{\psi(2 S) \rightarrow \mu^{+} \mu^{-}}=9.4 \pm 0.9 \pm 0.6 \pm 0.4 \mathrm{pb}$ for 7 and $13 \mathrm{TeV}$ collision energies, respectively $[5,6]$. The first uncertainty is statistical, the second is systematic and the third is due to the luminosity determination.

In addition, the cross-section times branching fraction to two muons is determined in bins of meson rapidity, y. The differential distributions are presented in Fig. 3 for $\sqrt{s}=7$ and $8 \mathrm{TeV}$. The measurements are compared to leading (LO) and next-to-leading order (NLO) predictions [7, 8, 9]. Better agreement is observed between data and the NLO prediction than LO one for results obtained for two different energies.

A model-dependent determination of photo-production cross-section, $\sigma(\gamma p \rightarrow \mathrm{J} / \psi p)$ is obtained from the LHCb differential cross-section measurement, assuming the power-law result from a fit to the HERA data [10] as shown in Fig. 4.

\subsection{Uspilon resonanses}

LHCb studied CEP of $\Upsilon(n S)$ states, where the resonances decay to the $\mu^{+} \mu^{-}$final state [11]. For the $\mu^{+} \mu^{-}$final state both muons lie in the pseudorapidity range $2<\eta\left(\mu^{ \pm}\right)<4.5$ and the $\Upsilon(n S)$ candidate is reconstructed in the rapidity range $2<y(\Upsilon(n S))<4.5$. The data sample used corresponds to an integrated luminosity of about $3 \mathrm{fb}^{-1}$ collected at $\sqrt{s}=7$ and $8 \mathrm{TeV}$, cf. Tab.1.

The determination of the exclusive signal yield has been performed by fitting the $\Upsilon(n S)$ invariant mass spectrum in order to separate $\Upsilon$ resonances from dimuon continuum background (left plot 

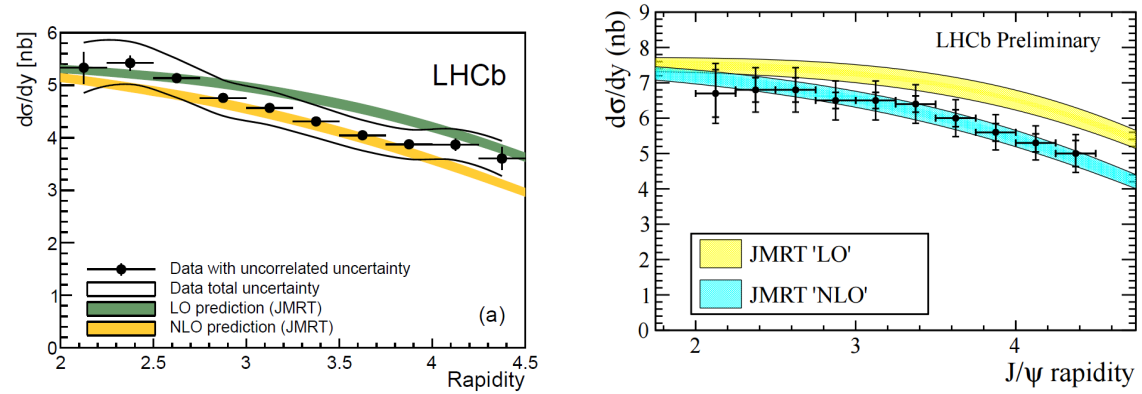

Figure 3: Differential cross-section for central exclusive $\mathrm{J} / \psi$ production compared to LO and NLO predictions for $7 \mathrm{TeV}$ (left, from [5]) and $13 \mathrm{TeV}$ (right, from [6]).
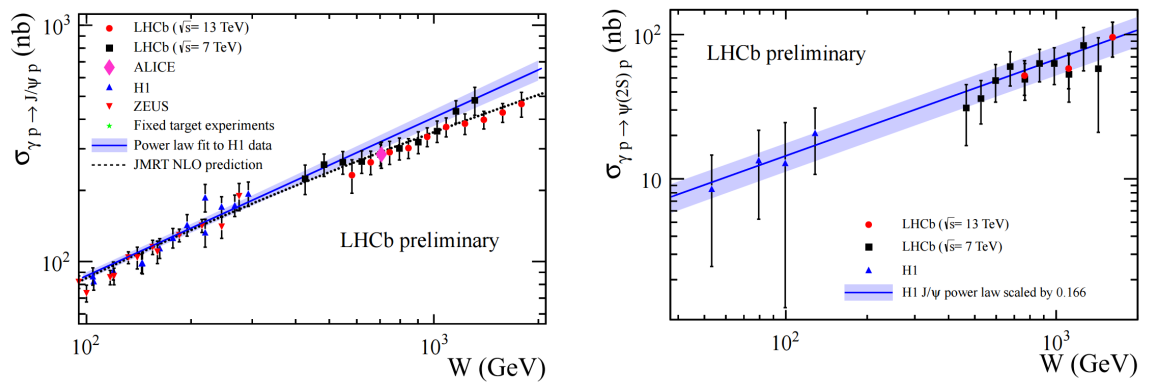

Figure 4: Compilation of photoproduction cross-section results for various experiments for $\mathrm{J} / \psi$ (left) and $\psi(2 S)$ (right) data, from [6].

in Fig.5) and by fitting the $\Upsilon(n S)$ candidate $p_{\mathrm{T}}^{2}$ distribution to distinguish exclusively produced $\Upsilon$ resonances from those originating in hard interactions (right plot in Fig.5).
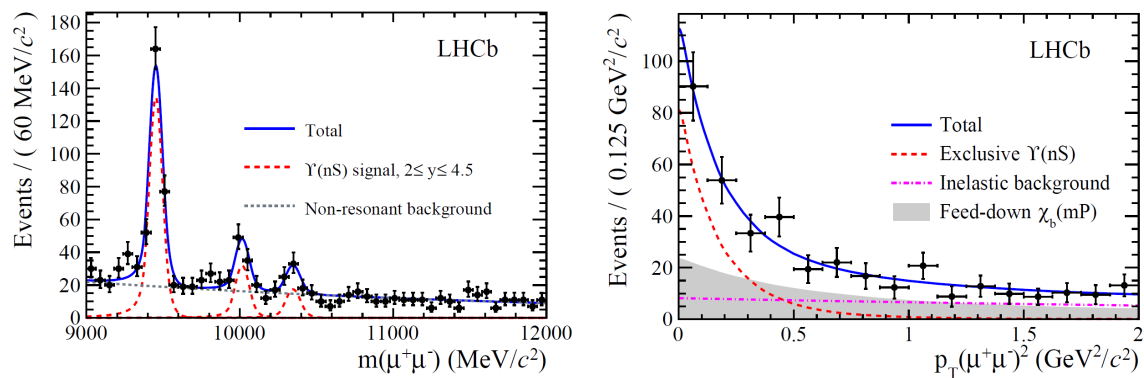

Figure 5: Left: Fit to the invariant dimuon mass spectrum in the rapidity range $2<y(\Upsilon)<4.5$ (black points). Right: Fit to the $p_{\mathrm{T}}^{2}$ distribution of the $\Upsilon$ candidates in the full rapidity range. For both fits compomonents are indicated in the legends. From [11].

Using the extracted signal fraction the measured $\Upsilon(1 S)$ and $\Upsilon(2 S)$ production cross-sections are $\sigma(p p \rightarrow p \Upsilon(1 S) p)=9.0 \pm 2.1 \pm 1.7 \mathrm{pb}$ and $\sigma(p p \rightarrow p \Upsilon(2 S) p)=1.3 \pm 0.8 \pm 0.3 \mathrm{pb}$, where the first uncertainties are statistical and the second are systematic. In addition the $\Upsilon(1 S)$ crosssection has also been measured as a function of rapidity and found to be in good agreement with SM predictions, cf. left plot in Fig.6. Finally, an upper limit has been set at $3.4 \mathrm{pb}$ at the $95 \%$ confidence level for the exclusive $\Upsilon(3 S)$ production cross-section, including possible contamination 
from $\chi_{b}(3 P) \rightarrow \Upsilon(3 S) \gamma$ decays.
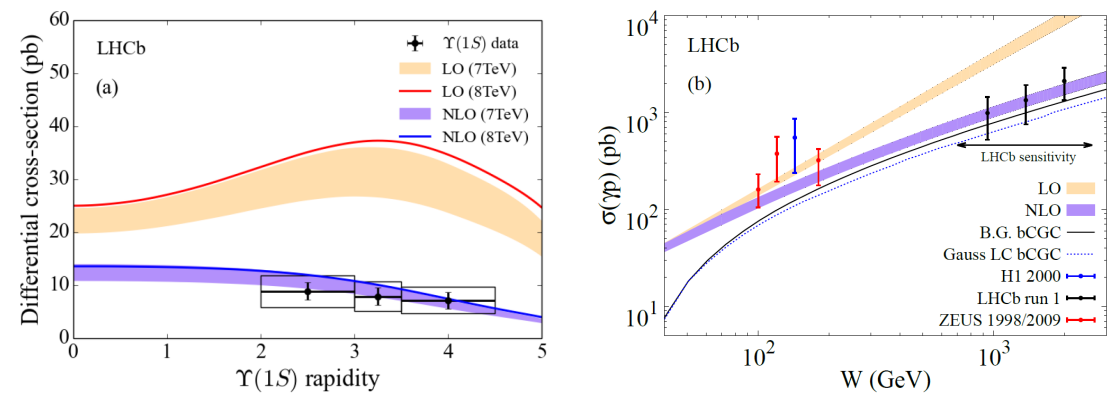

Figure 6: Measurements of exclusive $\Upsilon(1 S)$ photoproduction compared to theoretical predictions: (left) the $\Upsilon(1 S)$ cross-section in bins of rapidity, compared to LO and NLO predictions, (right) the photon-proton cross-sections extracted from the LHCb results compared to LO and NLO predictions and other experiments. From [11].

\subsection{Double charmonium}

CEP of $\mathrm{J} / \psi \mathrm{J} / \psi, \mathrm{J} / \psi \psi(2 S), \psi(2 S) \psi(2 S)$ have been studied by LHCb for which the dominant production mechanism is through double pomeron exchange [12]. The data sample used corresponds to an integrated luminosity of about $3 \mathrm{fb}^{-1}$ collected at $\sqrt{s}=7$ and $8 \mathrm{TeV}$, cf. Tab.1. The $\mathrm{J} / \psi$ and $\psi(2 S)$ mesons are identified through their decays to two muons. The selection of these mesons candidates requires four reconstructed tracks, three of them identified as muons, and no other activity in the event. The invariant masses of oppositely charged muon pairs is shown in the left plot of Fig.7. Accumulations of events are visible around the $\mathrm{J} / \psi$ and $\psi(2 S)$ masses. Similarly to the other analyses of this kind, the elastic fraction is estimated from the $p_{\mathrm{T}}^{2}$ distribution. It is shown in the right plot of Fig.7 for the $\mathrm{J} / \psi \mathrm{J} / \psi$ candidates. Central exclusive events peak below $1 \mathrm{GeV}^{2} / \mathrm{c}^{2}$ while the tail to higher values is characteristic of inelastic production.
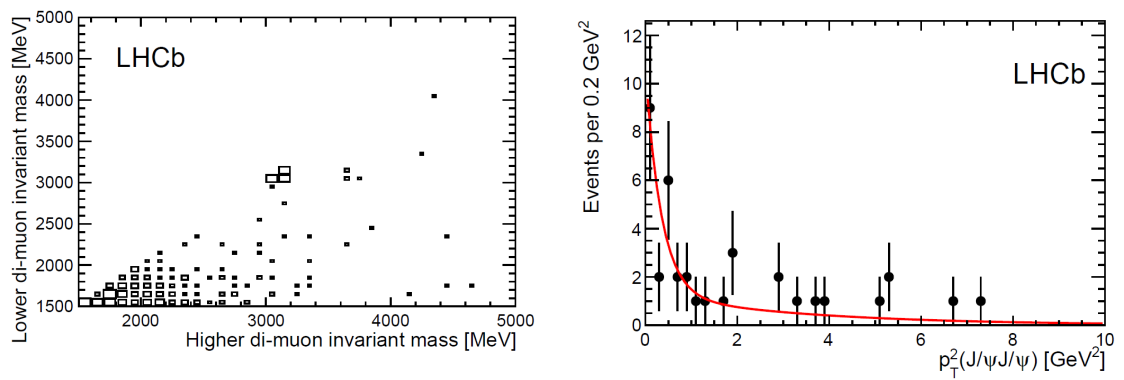

Figure 7: Left: Invariant masses of pairs of oppositely charged muons in events with exactly four tracks. Right: Fit to transverse momentum squared distribution of candidates for exclusively produced $\mathrm{J} / \psi \mathrm{J} / \psi$. From [12].

Using the extracted signal fraction the cross-sections for exclusive production of $\mathrm{J} / \psi \mathrm{J} / \psi$ and $\mathrm{J} / \psi \psi(2 S)$, pairs are measured. Searches have also been performed for pairs of P-wave charmonia and limits have been set on their production. The extracted cross-sections are: $\sigma(\mathrm{J} / \psi \mathrm{J} / \psi)=58 \pm$ 10 (stat) \pm 6 (syst)pb, and $\sigma(\mathrm{J} / \psi \psi(2 S))=63 \pm_{-18}^{+27}$ (stat) \pm 10 (syst)pb. The upper limits set at 
the $90 \%$ confidence level are found to be: $\sigma\left(\chi_{c 0} \chi_{c 0}\right)<0.069 \mathrm{pb}, \sigma\left(\chi_{c 1} \chi_{c 1}\right)<45 \mathrm{pb}, \sigma\left(\chi_{c 2} \chi_{c 2}\right)<$ $141 \mathrm{pb}[12]$.

\section{Summary and prospects}

A series of measurements of CEP has been performed by LHCb, dominantly based on data from Run 1. The results have been compared to theoretical predictions and those including NLO effects better describe the data. The first measurement of $\mathrm{J} / \psi$ and $\psi(2 S)$ meson production has been performed on Run 2 data following the installation of HeRSCheL instrumentation at high $\eta$, which allows for improved trigger efficiency for exclusive production processes and additional suppression of the background from inelastic interactions. Such techniques will be important for future analyses of exclusive production at $\mathrm{LHCb}$.

\section{Acknowledgement}

We acknowledge support from the national agencies: MNiSW and NCN (Poland) UMO-2015/18/M/ST2/00123.

\section{References}

[1] A. A. Alves, Jr. et al. [LHCb Collaboration], JINST 3 (2008) S08005. doi:10.1088/1748-0221/3/08/S08005

[2] K. Akiba et al. [LHC Forward Physics Working Group], J. Phys. G 43 (2016) 110201 doi:10.1088/0954-3899/43/11/110201 [arXiv:1611.05079 [hep-ph]].

[3] K. Akiba et al., LHCb-DP-2016-003.

[4] R. Aaij et al. [LHCb Collaboration], J. Phys. G 40 (2013) 045001 doi:10.1088/0954-3899/40/4/045001 [arXiv:1301.7084 [hep-ex]].

[5] R. Aaij et al. [LHCb Collaboration], J. Phys. G 41 (2014) 055002 doi:10.1088/0954-3899/41/5/055002 [arXiv:1401.3288 [hep-ex]].

[6] The LHCb Collaboration [LHCb Collaboration], LHCb-CONF-2016-007, CERN-LHCb-CONF-2016-007.

[7] S. P. Jones, A. D. Martin, M. G. Ryskin and T. Teubner, JHEP 1311 (2013) 085 doi:10.1007/JHEP11(2013)085 [arXiv:1307.7099 [hep-ph]].

[8] S. P. Jones, A. D. Martin, M. G. Ryskin and T. Teubner, J. Phys. G 43 (2016) no.3, 035002 doi:10.1088/0954-3899/43/3/035002 [arXiv:1507.06942 [hep-ph]].

[9] S. P. Jones, A. D. Martin, M. G. Ryskin and T. Teubner, J. Phys. G 41 (2014) 055009 doi:10.1088/0954-3899/41/5/055009 [arXiv:1312.6795 [hep-ph]].

[10] C. Alexa et al. [H1 Collaboration], Eur. Phys. J. C 73 (2013) no.6, 2466 doi:10.1140/epjc/s10052-013-2466-y [arXiv:1304.5162 [hep-ex]].

[11] R. Aaij et al. [LHCb Collaboration], JHEP 1509 (2015) 084 doi:10.1007/JHEP09(2015)084 [arXiv:1505.08139 [hep-ex]].

[12] R. Aaij et al. [LHCb Collaboration], J. Phys. G 41 (2014) no.11, 115002 doi:10.1088/0954-3899/41/11/115002 [arXiv:1407.5973 [hep-ex]]. 